\title{
Construcción de un índice de selección para rasgos de comportamiento en toros de lidia
}

\section{Building of a selection index for behavioral traits in fighting bulls}

Joel Domínguez-Viveros ${ }^{\text {a* }}$

Felipe Alonso Rodríguez-Almeida ${ }^{\mathrm{a}}$

Nicolás Callejas-Juárez ${ }^{\mathrm{a}}$,

Nelson Guadalupe Aguilar-Palma ${ }^{\mathrm{a}}$

Juan Ángel Ortega-Gutiérrez ${ }^{\mathrm{a}}$

${ }^{a}$ Universidad Autónoma de Chihuahua. Facultad de Zootecnia y Ecología. Periférico Francisco R. Almada, km 1. Chihuahua, Chih. México.

* Autor de correspondencia: joeldguezviveros@yahoo.com.mx

\section{- Resumen:}

En la ganadería de lidia, la selección está en función del desempeño del toro durante la lidia. Posición de la cabeza (PC) y de las manos (PM), recorrido del toro (RE) y modo de finalizar (FI) la embestida define el desempeño del toro, y pueden ser considerados como criterios de selección. El objetivo fue estimar heredabilidades $\left(\mathrm{h}^{2}\right)$ y correlaciones genéticas $\left(\mathrm{r}_{\mathrm{g}}\right)$ para PC, PM, FI y RE; y construir dos índices de selección: IS1 con valores relativos de un análisis factorial, IS2 con valores relativos a partir de la desviación estándar de cada rasgo. Se calculó la respuesta a la selección (RS) y exactitud (EX) de cada IS. El número de observaciones (valor promedio \pm desviación estándar) para PC, PM, RE y FI fue 984 (5.46 \pm 1.4), 978 (2.93 $\pm 1.1), 981(3.15 \pm 0.7)$ y $785(2.88 \pm 1.1)$, respectivamente; con escalas de uno a siete para 
PC, y de uno a cinco para el resto de las variables. Para $\mathrm{h}^{2}$ y $\mathrm{r}_{\mathrm{g}}$ se realizó un análisis multivariado con el software MTDFREML. La $\mathrm{h}^{2}$ fue de $0.20 \pm 0.06,0.15 \pm 0.05,0.13 \pm$ 0.03 y $0.17 \pm 0.05$ para PC, RE, PM y FI, respectivamente; todas las $r_{\mathrm{g}}$ fueron positivas, de 0.57 a 0.94 , con valor promedio de 0.753 . IS $1=0.068 * \mathrm{PC}+0.048^{*} \mathrm{RE}-0.012 * \mathrm{PM}+$ $0.088^{*} \mathrm{FI}$; con $\mathrm{EX}$ de 0.46 y $\mathrm{RS}$ de 0.325 . IS $2=0.231 * \mathrm{PC}+0.249 * \mathrm{RE}-0.038 * \mathrm{PM}+$ $0.298 * \mathrm{FI}$; con EX de 0.45 y RS de 1.15 .

- Palabras clave: Parámetros genéticos, Análisis multivariado, Mejoramiento genético, Respuesta selección.

\section{- Abstract:}

In Lidia cattle breed, the selection is based on the performance of the bull during the fighting. Head position (HP) and hands (PH), course of the bull (CB) and finish of the onslaught (FO), define the performance of the bull, and can be implemented as selection criteria. The objective was to estimate heritability $\left(\mathrm{h}^{2}\right)$ and genetic correlation $\left(\mathrm{r}_{\mathrm{g}}\right)$ for HP, PH, CB and FO; and building two selection indexes: IS1 with relative values of factorial analysis, IS2 by relative values from the standard deviation of each trait. The response to selection (RS) and accuracy (AC) were calculated. The number of observations (average \pm standard deviation) value for HP, PH, CB and FO were 984 (5.46 \pm 1.4$), 978$ (2.93 \pm 1.1$), 981$ (3.15 \pm 0.7$)$ and 785 (2.88 \pm 1.1$)$, respectively; with scales of one to seven for HP, and one to five for the rest of the variables. For $\mathrm{h}^{2}$ and $\mathrm{r}_{\mathrm{g}}$ a multivariate analysis was performed with MTDFREML software. The $\mathrm{h}^{2}$ was of $0.20 \pm 0.06,0.15 \pm 0.05,0.13 \pm 0.03$ y $0.17 \pm 0.05$ for HP, CB, PH y FO, respectively. All $r_{\mathrm{g}}$ were positive, from 0.57 to 0.94 , with average value of 0.753 . IS1 $=0.068 * \mathrm{HP}+0.048 * \mathrm{CB}-0.012 * \mathrm{PH}+0.088^{*} \mathrm{FO}$; with $\mathrm{AC}$ of 0.46 and $\mathrm{RS}$ of $0.325 . \mathrm{IS} 2=$ $0.231 * \mathrm{HP}+0.249 * \mathrm{CB}-0.038 * \mathrm{PH}+0.298 * \mathrm{FO}$; with AC of 0.45 and $\mathrm{RS}$ of 1.15 .

- Key words: Genetic parameters, Multivariate analysis, Genetic improvement, Selection response.

Recibido 11/10/2016

Aceptado 19/06/2017 


\section{Introducción V}

En la ganadería de lidia, los objetivos de selección están en función del desempeño del toro durante la lidia, valorando caracteres de comportamiento como agresividad y bravura, los cuales han diferenciado y caracterizado a esta raza de bovinos. A la lidia solo se envían los machos, y los criadores evalúan el desempeño del toro con dos notas de comportamiento: la lidia al caballo (LC), que se refiere a la embestida sobre el caballo y la respuesta a la pulla; y la lidia al torero (LT), que resume el comportamiento y desenvolvimiento con relación al torero ${ }^{(\mathbf{1})}$. Un toro bravo es aquel individuo que decide atacar o embestir al torero o al caballo, el cómo desarrollar el ataque o la agresión permite identificar o clasificar el nivel de bravura de esta raza de bovinos. Las notas de LC y LT son un resumen general del comportamiento del toro; sin embargo, existen otros rasgos que explican las diferencias en el desempeño del toro al realizar la agresión, la posición de la cabeza (PC) y posición de las manos (PM), definen la postura y el estilo del toro al realizar el ataque; el recorrido del toro (RE) sobre la muleta y el modo de finalizar (FI) la acometida, representan la movilidad y la emotividad del toro al embestir. Las cuatro variables (PC, PM, RE y FI) en su conjunto definen el desempeño del toro en función de la bravura y pueden ser implementadas como criterios de selección.

En el establecimiento de programas de mejoramiento genético, la definición de criterios de selección requiere cuantificar la proporción de los posibles efectos genéticos y ambientales, que inciden sobre la variabilidad total de la población ${ }^{(2)}$; del mismo modo, considerar dos o más características en los criterios de selección, requiere implementar un método de selección para caracteres múltiples, como es el caso del índice de selección ${ }^{(3)}$. El índice de selección permite la predicción de valores genéticos de los candidatos a selección, aplicando ponderaciones a los valores fenotípicos con propiedades de mejor predictor lineal, además: a) minimiza el error de predicción, b) maximiza la exactitud en las predicciones, así como la respuesta a la selección, y c) maximiza la probabilidad de ordenar correctamente a los animales con base en el arreglo de valor aditivo ${ }^{(4,5)}$. Los objetivos del presente estudio fueron: a) estimar parámetros genéticos para los rasgos de PC, PM, FI y RE; y, b) generar un índice de selección con base en los cuatro rasgos analizados.

\section{Material y métodos V}

La base de datos con la información genealógica y de variables de comportamiento fue proporcionada por la ganadería Los Encinos, ubicada en el estado de Querétaro; datos 
particulares del origen, conformación y sistema de producción, así como estudios previos sobre estructura del pedigrí y parámetros de poblaciones fueron publicados por Castillo ${ }^{(6)}$ y Domínguez-Viveros et $a l^{(7)}$. Se analizó la información de PC, PM, RE y FI; durante la lidia el ganadero evaluó el desempeño del toro y al final asignó una anotación para cada uno de los rasgos, con escalas de uno a siete para PC, y de uno a cinco para el resto de las variables, concediendo el valor más alto al mejor. El número de observaciones (valor promedio \pm

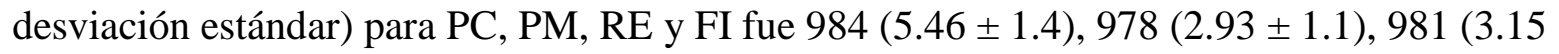
$\pm 0.7)$ y 785 (2.88 \pm 1.1$)$, respectivamente; los toros con información de comportamiento son nacidos de 1994 a 2013, y las valoraciones se realizaron por un solo evaluador. Posterior al proceso de edición, con la prueba estadística de Kolmogorov se verificó que las variables analizadas cumplieran con las especificaciones de normalidad; además, se realizaron análisis de varianza para caracterizar a la población en función de los efectos ambientales y definir la estructura de grupos contemporáneos ${ }^{(1,8,9)}$; los análisis se realizaron con los procedimientos UNIVARIATE y GLM, del programa para análisis estadístico SAS ${ }^{(\mathbf{1 0})}$.

Para la estimación de parámetros genéticos, se realizó un análisis multivariado con las cuatro variables, a partir del modelo mixto: $\boldsymbol{y}=\boldsymbol{X} \boldsymbol{b}+\boldsymbol{Z} \boldsymbol{a}+\boldsymbol{e}$; donde

y es el vector de registros;

$\boldsymbol{b}$ es el vector de efectos fijos, que incluyó los grupos contemporáneos definidos por la combinación de año y época de nacimiento, más la covariable de edad del individuo a la lidia en función lineal y cuadrática;

$\boldsymbol{a}$ es el vector de efectos aleatorios, genéticos aditivos directos;

$\boldsymbol{e}$ es el vector de efectos residuales aleatorios;

$\boldsymbol{X}$ y $\boldsymbol{Z}$ son matrices de incidencia que relacionan a las observaciones en $\mathbf{y}$ con los respectivos efectos. El arreglo matricial para el análisis multivariado quedó definido como: $\left[\begin{array}{l}y_{i} \\ y_{j}\end{array}\right]=$ $\left[\begin{array}{cc}x_{i} & 0 \\ 0 & x_{j}\end{array}\right]\left[\begin{array}{l}b_{i} \\ b_{j}\end{array}\right]+\left[\begin{array}{cc}z_{i} & 0 \\ 0 & z_{j}\end{array}\right]\left[\begin{array}{l}a_{i} \\ a_{j}\end{array}\right]+\left[\begin{array}{l}e_{i} \\ e_{j}\end{array}\right]$. La dimensión y estructura de las matrices $\boldsymbol{X}$ y $\boldsymbol{Z}$ estuvo en función del número de individuos $(\mathrm{N})$ con datos de comportamiento para cada variable analizada; las suposiciones del modelo fueron: $\boldsymbol{E}[\boldsymbol{y}]=\boldsymbol{X} \boldsymbol{\beta}, \boldsymbol{E}[\boldsymbol{a}]=\mathbf{0}$ y $\boldsymbol{E}[\boldsymbol{e}]=\mathbf{0}$, y la estructura de varianzas y covarianzas quedo de la siguiente forma: $\operatorname{Var}\left[\begin{array}{c}a_{i} \\ a_{j} \\ e_{i} \\ e_{j}\end{array}\right]=\left[\begin{array}{cccc}A \sigma_{a i}^{2} & A \sigma_{a i a j} & 0 & 0 \\ A \sigma_{a i a j} & A \sigma_{a j}^{2} & 0 & 0 \\ 0 & 0 & I_{N} \sigma_{e i}^{2} & \sigma_{e i e j} \\ 0 & 0 & \sigma_{e i e j} & I_{N} \sigma_{e j}^{2}\end{array}\right]$, donde: $\boldsymbol{A}$, es la matriz con todas las relaciones genéticas aditivas (parentesco) entre todos los animales en el pedigrí; $\boldsymbol{\sigma}_{a i}^{2}=$ varianzas de efectos genéticos aditivos directos; $\boldsymbol{\sigma}_{a i a j}=$ covarianzas entre efectos genéticos aditivos directos; $\sigma_{e i}^{2}=$ varianzas residuales; $\sigma_{e i e j}=$ covarianzas entre efectos residuales. El pedigrí estuvo conformado por 8019 individuos 
nacidos de 1964 a 2015. Con el procedimiento de máxima verosimilitud restringida, y libre de derivadas, se estimó las (co)varianzas genéticas y fenotípicas $\left(\boldsymbol{\sigma}_{p}^{2}\right)$; así como la heredabilidad $\left(\mathrm{h}^{2}=\sigma_{a}^{2} / \sigma_{p}^{2}\right)$ para cada variable, y las correlaciones genéticas $\left(\mathrm{r}_{\mathrm{g}}=\sigma_{a i a j} /\right.$ $\left.\sigma_{a i} * \sigma_{a j}\right)$ a través de las cuatro variables de estudio; el análisis se realizó con el software MTDFREML ${ }^{(11)}$.

Para la construcción del índice de selección (IS), el objetivo de selección $(\boldsymbol{H})$ está definido por una función lineal de los valores genético aditivos $(\boldsymbol{g})$, ponderados por los valores económicos o relativos $(\boldsymbol{a})$ de las características consideradas en los criterios de selección: $\boldsymbol{H}$ $=\boldsymbol{a}_{1} \boldsymbol{g}_{\boldsymbol{1}}+\boldsymbol{a}_{2} \boldsymbol{g}_{2}+\boldsymbol{a}_{3} \boldsymbol{g}_{3}+\boldsymbol{a}_{4} \boldsymbol{g}_{4}$. El IS se definió como una función lineal de $\boldsymbol{I}=\boldsymbol{b}_{1} \boldsymbol{x}_{\boldsymbol{1}}+\boldsymbol{b}_{2} \boldsymbol{x}_{2}+$ $b_{3} x_{3}+b_{4} x_{4}$; la estimación de los $b_{i}$ se realizó a partir de ${ }^{(4,5)} \boldsymbol{P b}=\boldsymbol{G} \boldsymbol{a}$, y se resolvió como $\boldsymbol{b}=$ $\boldsymbol{P}^{-1} \boldsymbol{G} \boldsymbol{a}$, donde: $\boldsymbol{P}=$ matriz simétrica con la estructura de varianzas (en la diagonal) y covarianzas (fuera de la diagonal) fenotípicas; $\boldsymbol{G}=$ matriz simétrica de (co)varianzas genéticas; $\boldsymbol{b}=$ vector con los coeficientes de regresión múltiple para cada característica; $\boldsymbol{x}_{\boldsymbol{i}}=$ valor fenotípico de la i-ésima característica considerada en el criterio de selección, expresada como una desviación de su media; $\boldsymbol{a}=$ vector de valores relativos $(\mathrm{VR})$ o de proporción, los cuales fueron calculados en dos modalidades: VR1 = valores relativos estimados a través de un análisis de factorial (AF); y, VR2 = valores relativos asignados a una desviación estándar del cambio de cada carácter, específicamente, los pesos fueron los recíprocos $\left(1 / \sigma_{p}=\sigma_{p} /\right.$ $\sigma_{p}^{2}$ ) de la desviación estándar fenotípica ${ }^{(5,12)}$.

El $\mathrm{AF}^{(\mathbf{1 3}, \mathbf{1 4})}$, a través de la matriz correlaciones, tiende a explicar una variable observada $(\boldsymbol{Z})$ por un número de variables latentes, o no observadas, que se denominan factores $(\boldsymbol{F})$; en el modelo de análisis factorial, cada variable $\boldsymbol{Z}$ será una combinación de los $\boldsymbol{F}$ más de los errores $(\boldsymbol{e})$. La variable $\boldsymbol{Z}$ define el comportamiento del toro durante la lidia, los $\boldsymbol{F}$ son los rasgos analizados, y el AF se realizó con la matriz de correlaciones fenotípicas. Los rasgos analizados $(\boldsymbol{F})$ se encuentran correlacionados, dado que comparten algo en común, y algo que los diferencia; las diferencias en $\boldsymbol{Z}$ se deben a $\operatorname{los} \boldsymbol{F}$ que comparten (comunalidad) y a los factores específicos o propios (especificidad). En términos de varianzas, la varianza en $\boldsymbol{Z}$ se debe a la varianza de comunalidad más la variabilidad especifica; el vector de valores relativos $(\boldsymbol{a})$ se obtuvo a partir de los elementos de la varianza de comunalidad, los cuales se ajustaron a términos de proporción de la suma total; el valor a proporción de cada rasgo, expresó cuánto de la varianza común en $\boldsymbol{Z}$ es explicado por el rasgo en particular.

Con los vectores de valores relativos, se generaron dos índices de selección: IS1 para VR1, e IS2 para VR2. Se estimó la varianza de cada IS ${ }^{(15,16,17)}\left(\sigma_{I S}^{2}=\boldsymbol{b}^{\prime} \boldsymbol{P b}\right)$ y del H $\left(\sigma_{\boldsymbol{H}}^{2}=\boldsymbol{a}^{\prime} \boldsymbol{G a}\right)$, así como las covarianzas $\left(\sigma_{I S, \boldsymbol{H}}=\boldsymbol{b}^{\prime} \boldsymbol{G a}\right)$ y las correlaciones: $\boldsymbol{r}_{I S, \boldsymbol{H}}=\sigma_{I S, \boldsymbol{H}} / \sigma_{I S}{ }^{*} \sigma_{\boldsymbol{H}}=\boldsymbol{b}^{\prime} \boldsymbol{G} \boldsymbol{a}$ / $\left(\boldsymbol{b}^{\prime} \boldsymbol{P b}\right)^{0.5}\left(\boldsymbol{a}^{\prime} \boldsymbol{G a}\right)^{0.5}$. La correlación $\boldsymbol{r}_{I S, H}$ establece la exactitud del IS, y permitió estimar la respuesta a la selección, $S_{H}=i * r_{I S, H} * \sigma_{H}$; donde $i$ es la intensidad de selección, y $\sigma_{H}=\left(\sigma_{H}^{2}\right)^{0.5}$; para $\boldsymbol{i}$ se utilizó el valor promedio de 1.735, a partir de una presión de selección del $50 \%$ en vaquillas y del $1 \%$ en machos, dado las características de la ganadería de toros de lidia $^{(7)}$. 
Los análisis del AF y las operaciones con matrices del IS se realizaron con los procedimientos FACTOR e IML, del programa para análisis estadísticos $S A S^{(10)}$.

\section{Resultados y discusión V}

En el Cuadro 1 se presentan los resultados para las $\mathrm{h}^{2}$ y $\mathrm{r}_{\mathrm{g}}$ a través de las cuatro variables analizadas; así como los VR1 y VR2 utilizados en los IS. La $\mathrm{h}^{2}$ promedio fue de 0.163 , con un valor mínimo de $0.13 \pm 0.03$; las $\mathrm{h}^{2}$ pueden ubicarse como de medio a bajo índice de herencia, como criterio de selección la respuesta a la selección va a ser muy baja, por lo que debe acompañarse de características con valores de índice de herencia de medio a alto, y sus valores de correlación genética, para mejorar el pronóstico. Todas las $r_{g}$ fueron positivas con un valor promedio de 0.753 , en un rango de $0.57 \pm 0.11$ a $0.94 \pm 0.18$; estos rasgos que determinan el comportamiento del toro en la lidia están asociadas genéticamente, y la posible respuesta a la selección correlacionada, o de manera conjunta, sería en el mismo sentido. Los IS construidos fueron: IS $1=0.068 * \mathrm{PC}+0.048 * \mathrm{RE}-0.012 * \mathrm{PM}+0.088 * \mathrm{FI}$, con los componentes: $\sigma_{I S}^{2}=0.033, \sigma_{H}^{2}=0.161, \boldsymbol{r}_{I S, \boldsymbol{H}}=0.46, \mathrm{y} \boldsymbol{S}_{\boldsymbol{H}}=0.325$. De igual forma, IS2 $=$ $0.231 * \mathrm{PC}+0.249 * \mathrm{RE}-0.038 * \mathrm{PM}+0.298 * \mathrm{FI}$, con sus componentes: $\sigma_{I S}^{2}=0.446, \sigma_{H}^{2}=$ $2.17, \boldsymbol{r}_{I S, \boldsymbol{H}}=0.45, \mathrm{y} \boldsymbol{S}_{\boldsymbol{H}}=1.15$.

Cuadro 1: Valores relativos y parámetros genéticos para los rasgos que determinan el comportamiento del toro de lidia

\begin{tabular}{lccccccc}
\hline & \multicolumn{3}{c}{ Parámetros genéticos } & \multicolumn{3}{c}{ Valores relativos } \\
& PC & RE & PM & FI & FC & VR1 & VR2 \\
\hline PC & $0.20 \pm 0.06$ & & & & 0.834 & 0.24 & 0.714 \\
RE & $0.57 \pm 0.11$ & $0.15 \pm 0.05$ & & & 0.785 & 0.23 & 1.428 \\
PM & $0.69 \pm 0.13$ & $0.72 \pm 0.15$ & $0.13 \pm 0.03$ & & 0.899 & 0.26 & 0.909 \\
FI & $0.90 \pm 0.18$ & $0.70 \pm 0.16$ & $0.94 \pm 0.18$ & $0.17 \pm 0.05$ & 0.916 & 0.27 & 0.919 \\
\hline
\end{tabular}

$\mathrm{PC}=$ posición de la cabeza, $\mathrm{RE}=$ recorrido, $\mathrm{PM}=$ posición de las manos, $\mathrm{F}=$ finalización. Estimaciones de heredabilidad ( \pm error estándar) en la diagonal, y de correlaciones genéticas bajo la diagonal. $\mathrm{FC}=$ vector resultado del análisis factorial. VR1= vector de valores a proporción, a partir del análisis factorial. VR2= vector de valores relativos, producto del reciproco de la desviación estándar fenotípica $\left(1 / \sigma_{p}\right)$. 
En estudios afines, Pelayo et $a l^{(18)}$ estimaron $\mathrm{h}^{2}$ en un intervalo de 0.13 a 0.41 , con valor promedio de 0.29 , para doce variables asociadas al desempeño del toro; además, el $93.9 \%$ de las $r_{\mathrm{g}}$ fueron positivas en el intervalo de 0.01 a 0.90 , las $\mathrm{r}_{\mathrm{g}}$ negativas $(6.1 \%)$ oscilaron entre -0.17 y -0.38. Asimismo, con un análisis multivariado (correlaciones canónicas) reagruparon las doce variables analizadas a través de tres índices de selección, en función de tres criterios de selección: agresividad, fuerza de ataque y movilidad. En España ${ }^{(19)}$ analizaron catorce variables relacionadas con el comportamiento de los toros durante la lidia, y estimaron $\mathrm{h}^{2}$ en un intervalo de 0.08 a 0.35 , con un promedio general de 0.25 ; de la misma forma, Silva et $a l^{(20)}$ para variables asociadas a la lidia como agresividad, ferocidad y movilidad, publicaron estimaciones de $\mathrm{h}^{2}$ en un intervalo de 0.28 a 0.36 . En la ganadería de lidia mexicana las notas de LC y LT son los principales criterios de selección; DomínguezViveros et $a l^{(1)}$ publicaron $\mathrm{h}^{2}$ de 0.09 para LC y 0.18 para LT, para la ganadería del presente estudio; de igual forma, reportaron $\mathrm{h}^{2}$ en un intervalo de 0.16 a 0.47 para LC, y de 0.22 a 41 para LT, de otras tres ganaderías mexicanas de lidia.

En su contexto, el IS maximiza la exactitud en las predicciones, así como la respuesta a la selección $^{(4,5)}$; la exactitud del IS1 e IS2 fueron similares; las diferencias en respuesta a la selección ( 0.325 vs 1.15$)$ se atribuyen a la estructura y efectos de los valores relativos (VR1 y VR2) sobre la desviación estándar del índice $\left(\sigma_{H}\right)$. En la ganadería de lidia el proceso de selección se realiza en la tienta; los toros evaluados durante la lidia aportan información que se utiliza para la selección; sin embargo, la lidia finaliza con la muerte del toro. La tienta es una prueba de comportamiento similar a la lidia, sin sacrificio del animal y se realiza alrededor de los dos años de edad; en esta prueba se evalúa el comportamiento del animal y la expresión de los rasgos que determinan la calidad y el desarrollo de la lidia. En machos la selección se enfoca en tres aspectos: prospectos a semental, clasificación de los toros para los diferentes festejos o corridas, y desechos; en hembras, la selección se enfoca en vaquillas para reemplazo y desechos. Con el IS, el criador podrá jerarquizar a los prospectos en función de los tres aspectos de selección, con base en el valor genético aditivo, y maximizando la probabilidad de ordenar correctamente a los animales con arreglo a su valor aditivo en función de los cuatro rasgos ${ }^{(15)}$.

Un IS, es una herramienta muy específica de una ganadería de lidia; el IS se realiza en función de los objetivos de selección, sus características y cualidades dependen de la estructura, calidad y cantidad de información genealógica y de comportamiento, de los parámetros genéticos asociados a los criterios de selección, así como la importancia relativa o porcentual de cada criterio de selección, entre otras variantes ${ }^{(21,22)}$. La apreciación, clasificación y valoración de los objetivos de selección difieren a través de ganadería; un IS es estricto dentro de ganadería, cada criador o evaluador tiene una apreciación diferente del buen comportamiento o calidad del desempeño del toro durante la lidia. El IS permite separar individuos con base en la evaluación simultánea de varios caracteres; cada IS tiene una eficiencia diferente, depende de las características que incluya, sus valores relativos, la 
variabilidad genética y fenotípica y sus valores de correlaciones entre ellas, el IS que proporcione la ganancia genética máxima por unidad de tiempo es el mejor ${ }^{(23)}$.

El toro de lidia como raza especializada, posee su patrón racial denominado "trapío", y las características que definen la bravura (en lo particular y correlacionadas) están asociadas al desempeño durante la lidia, que a través del tiempo le han ido delimitando y atribuyendo los criadores; estos sistemas de producción y las características de su mercado, dado las diversas plazas o festejos donde van los toros a la lidia, tienen sus contrastes que deben considerarse al establecer un programa de mejoramiento genético con base en selección. Sánchez et al ${ }^{(\mathbf{2 4})}$ calificaron el comportamiento del toro en la lidia a través de 21 rasgos específicos, y posterior a un análisis multivariado con base en componentes principales, definieron el desempeño del toro a través de cuatro componentes: decisión de ataque o bravura en la muleta; decisión o bravura al caballo; calidad de la embestida a la muleta; postura y progreso de la lidia.

\section{"Conclusiones e implicaciones \|}

Las heredabilidades para los rasgos analizados quedaron en el intervalo de 0.13 a 0.20 ; aunque de mediana a baja magnitud, indica que presentan variabilidad genética y pueden ser considerados como criterios de selección. Todas las correlaciones genéticas fueron positivas, en el intervalo de 0.57 a 0.94 . Los rasgos evaluados están asociados genéticamente, y la posible respuesta a la selección correlacionada sería en el mismo sentido. Se generaron dos índices de selección, en función al procedimiento de estimación del vector de valores relativos; la exactitud del índice fue similar en ambos casos (0.45); sin embargo, la respuesta a la selección fue superior en el índice de selección generado con valores relativos a partir de la desviación estándar fenotípica.

\section{N Agradecimientos}

Se agradece al propietario de la ganadería Los Encinos, el proporcionar la información genealógica y de comportamiento para el desarrollo del presente estudio. 


\section{- Literatura citada:}

1. Domínguez-Viveros J, Rodríguez-Almeida FA, Núnez-Domínguez R, RamírezValverde R, Ruiz-Flores A. Parámetros genéticos y tendencias genéticas para características de comportamiento en ganaderías de lidia mexicanas. Rev Mex Cienc Pecu 2014;5:261-271.

2. Delgado JV, Molina A, Camacho ME, Rodero A. Evolución de la metodología de la mejora genética en el vacuno de carne. Arch Zootec 1995;44:111-121.

3. Bourdon RM. Understanding animal breeding. First ed. New Jersey, USA: Prentice Hall; 1997.

4. Van Vleck D. Selection index and introduction to mixed model methods. First ed. Florida, USA: CRC Press; 1993.

5. Cameron, ND. Selection indices and prediction of genetic merit in animal breeding. First ed. Edinburgh, UK: CAB International; 1997.

6. Castillo GE. Nuestro toro. Asociación Nacional de Criadores de Toros de Lidia. México, DF. 2003.

7. Domínguez-Viveros J, Rodríguez-Almeida FA, Núñez-Domínguez R, RamírezValverde R, Ortega-Gutiérrez JA, Ruiz-Flores A. Análisis del pedigrí y efectos de la consanguinidad en el comportamiento del ganado de lidia mexicano. Arch Zootec 2010;59:63-72.

8. Robinson GK. That BLUP is a good thing: the estimation of random effects. Statist Sci 1991;6:15-32.

9. Gianola D. Statistic in animal breeding. J American Statis Assoc 2000;95:296-299.

10. SAS. SAS/STAT User's Guide (Release 9.0). Cary NC, USA: SAS Inst. Inc. 2005.

11. Boldman KG, Kriese LA, Van Vleck LD, Van Tassell CP, Kachman SD. A Manual for use of MTDFREML. A set of programs to obtain estimates of variances and covariances (Draft). USDA. ARS. 1995.

12. Atil H. A comparison of different selection indices for genetic improvement for milk traits in Holstein Friesian cattle in Turkey by using one standard deviation as relative economic weight. Pak J Biol Sci 2006;9:285-288. 
13. Hair JF, Anderson RE, Tatham RL, Black WC. Multivariate data analysis. First ed. Madrid, España: Prentice Hall International, Inc; 1999.

14. Peña D. Análisis de datos multivariantes. Madrid, España: Editorial McGraw Hill; 2002.

15. Hazel LN, Dickerson GE, Freeman D. The selection index - then, now and for the future. J Dairy Sci 1994;77:3236-3251.

16. Lin CY. An iterative procedure for deriving selection indexes with constant restrictions. J Anim Sci 2005;83:2313-2318.

17. Falconer DS, Mackay TFC. An Introduction to quantitative genetics. 4th ed. London, UK: Longman Pub; 1996.

18. Pelayo R, Solé M, Sánchez MJ, Molina A, Varela M. Behavioural linear standardized scoring system of the Lidia cattle breed by testing in herd: estimation of genetic parameters. J Anim Breed Genet 2016;133:414-421.

19. Silva B, Gonzalo A, Cañón J. Genetic parameters of behavior traits in the bovine (Bos taurus). Proc. $7^{\text {th }}$ Wld Congr Genet Appl Livest Prod. Montpellier, France. 2002.

20. Silva B, Gonzalo A, Cañón J. Genetic parameters of aggressiveness, ferocity and mobility in the fighting bull breed. Anim Res 2006;55:65-70.

21. Vergara GO, Iriarte MC. Determinación de un índice de selección para el peso al nacer y al destete en ganado Cebú. Rev MVZ Córdoba 2002;7:148-151.

22. Montes VD, Vergara GO, Prieto ME. Determinación de un índice de selección para el peso al nacer y al destete en ganado bovino de la raza Brahman. Rev MVZ Córdoba 2008; $13: 1365-1368$.

23. Cerón-Rojas JJ, Sahagún-Castellanos J. Un índice de selección basado en componentes principales. Agrociencia 2005;39:667-677.

24. Sánchez JM, Riol JA, Castro MJ, Gaudioso VR. Comportamiento del toro de lidia frente al caballo y muleta: aspectos aplicativos en la selección de la raza. Arch Zootec 1990;39:165-174. 